\title{
GAMPI
}

\section{Movimentação e Tranferência de Pacientes no leito: Uma questão para o Design}

\author{
Moving and Transfer Patients in bed: A design issue \\ ALVES, Marcelo; Especialista; Univille \\ marcelo.alves2702@gmail.com
}

CAVALCANTI, Anna; Mestre; Univille anna.cavalcanti08@gmail.com

\section{Resumo}

Este artigo refere-se a pesquisa em andamento no Mestrado Profissional em Design, relatando os problemas relacionados às pessoas com mobilidade reduzida, destacando-se os idosos. A fragilização do idoso cria implicações para os sistemas de cuidados, demandando a assistência de um cuidador para a realização das atividades cotidianas. Esta pesquisa embasa a criação de uma solução por meio do design, para a atividade de movimentação e transferência das pessoas com mobilidade reduzida no leito. Foram realizadas pesquisas bibliográficas acerca do tema e uma pesquisa de campo em duas Instituições de Longa Permanência-ILP na cidade de Joinville, Santa Catarina.

Palavras Chave: Mobilidade reduzida; Design; Tecnologia; Movimentação e Transferência.

\section{Abstract}

This article refers to ongoing research in the Professional Masters, reporting problems related to people with reduced mobility, highlighting the elderly people. The elderly weakening creates implications for for care systems, requiring the assistance of a caregiver to carry out daily activities. This research underpins the creation of a solution through design for drive activity and transfer of persons with reduced mobility in bed. Literature searches were conducted on the subject and a field survey in two long stay institutions in the city of Joinville, Santa Catarina.

Keywords: reduced mobility; design; technology; handling and transfer. 


\section{GAMPII $^{\text {GA }}$}

\section{INTRODUÇÃO}

Apesar da abundância de tecnologias disponíveis para a área da saúde, ainda existem enormes lacunas na disponibilidade, associada a custos acessíveis e que sejam seguras, eficazes e apropriadas para o profissional da saúde, incluindo a mais básica das tecnologias de assistência necessária para atender às necessidades das pessoas idosas (WHO-Alex Ross, 2013).

O foco desta pesquisa são as pessoas com mobilidade reduzida. Neste cenário, destacam-se os idosos, os quais representam uma população crescente no país, trazendo implicações para a sociedade que podem afetar os sistemas de cuidados, com o aumento do número total de casos de doenças crônicas e degenerativas, consequentemente provocando a diminuição da capacidade funcional, tornando o idoso frágil, comprometendo sua mobilidade e capacidade de executar as atividades da vida diária, influenciando na sua qualidade de vida e bem-estar (CHRISTOPHE, 2007). Desta maneira serão exigidas novas habilidades de profissionais da área de saúde, das indústrias, dos designers e da sociedade de um modo geral, para garantir as condições necessárias de cuidado.

As instituições de longa permanência (ILPs), sejam elas públicas ou privadas, podem ser uma alternativa de cuidado de longa duração, gerando um benefício social na garantia da qualidade de vida dos idosos nas últimas fases da vida e de pessoas que precisam de cuidados especiais.

Este cenário motivou a presente pesquisa aplicada, com o objetivo estudar as questões acerca do envelhecimento populacional e o ato de cuidar nas ILPIs, com foco na atividade de movimentação e transferência das pessoas com mobilidade reduzida no leito, procurando compreender a relação dos cuidadores e idosos, observando as necessidades destes usuários frente as dificuldades dos mesmos no dia a dia.

A metodologia de pesquisa foi baseada no processo do Design Thinking, que possui as seguintes fases: Empatia, Definição, Criação, Prototipagem e Teste. As informações descritas servirão como embasamento para o desenvolvimento de uma tecnologia para solucionar o problema proposto. 


\section{GAMPI plural'15}

\section{CONTEXTUALIZAÇÃO}

De acordo com o documento Development in an Ageing World publicado pela Nações Unidas (UN), o ambiente social em que as pessoas envelhecem está mudando rapidamente, trazendo implicações importantes nos países desenvolvidos e em desenvolvimento. O tamanho das famílias está diminuindo, as percepções em relação ao apoio, carinho e as condições das pessoas mais velhas, vem se transformando. Nesse cenário, faz-se necessário a expansão do fornecimento de cuidados formais de longo prazo para as pessoas idosas, incluindo vida institucional (serviço formal), bem como os serviços alternativos para aqueles que desejarem ficar em suas casas (serviço informal).

O Brasil é um dos países em desenvolvimento em que o ritmo de envelhecimento é acelerado, haja vista que o estatuto do idoso foi implantado somente em 2003 sob a Lei 10.741/2003. Segundo o estatuto, uma pessoa é considerada idosa a partir de 60 anos de idade. O país ainda galga, a passos lentos, a criação de uma legislação forte que realmente garanta uma estrutura social que provenha os recursos necessários para garantir o envelhecimento com dignidade e bem-estar de sua população.

Em 2014, segundo o IBGE, no Brasil o número de idosos era de 20,6 milhões, representando $10,8 \%$ da população total. Destes, $7,9 \%$ são pessoas com 65 anos ou mais e as projeções indicam que a estimativa de vida passará de 75 anos em 2013, para 81 em 2060.

Camarano e Kanso (2010) demonstram um cenário para 2020 em que 4,5 milhões de idosos terão dificuldades nas atividades da vida diária, e necessitarão de cuidados de longa duração. Este número é cerca de 1,3 milhão a mais comparado com 2008, e deste total, as mulheres representam cerca de $62,7 \%$. No entanto se houver melhorias nas condições de saúde e qualidade de vida, a quantidade de idosos com incapacidade funcional que necessitarão de cuidados de longa duração em 2020, cairá para 3,8 milhões. Neste cenário as mulheres também serão predominantes.

Outro cenário apontado por Camarano e Kanso (2010) é a projeção para 2020 do número de idosos que não estarão sob o cuidado familiar, estimado em torno de 2 milhões. Este dado pode realçar a necessidade de um aumento da quantidade dos cuidados formais que poderão ser ofertados pelas ILPIs. 


\section{GAMPI
plural'}

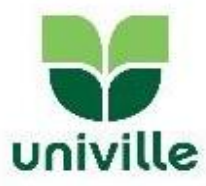

Segundo Christophe (2007), a gerontologia e a geriatria apontam que o envelhecimento traz doenças crônicas, degenerativas e a diminuição da capacidade funcional, tornando o idoso frágil, comprometendo sua mobilidade e qualidade de vida. As definições de capacidade funcional seguem a Organização Mundial de Saúde - OMS, que as relaciona aos conceitos de autonomia, independência, qualidade e expectativa de vida saudável. $\mathrm{O}$ autônomo é aquele que possui a capacidade de tomar decisões sobre a sua vida diária de acordo com a sua consciência. $\mathrm{O}$ independente é o indivíduo que possui a capacidade de viver independente com alguma ou nenhuma ajuda. As atividades são classificadas pela OMS como AVDs (atividades da vida diária - tomar banho, comer, usar o banheiro, deambulação pelos cômodos, etc.) e AIVDs (atividades instrumentais da vida diária - trabalhos domésticos, fazer compras, preparar as refeições, etc.). A perda da capacidade funcional é determinada através de graus de dependência e pode ser gradual, definitiva ou reversível (Camarano e Mello, 2010).

A RDC n 283, de 26 de setembro de 2005, define três graus de dependência. O grau de dependência I são idosos independentes, mesmo que necessitem de equipamentos de autoajuda, o grau de dependência II são os idosos com dependência de até três atividades de autocuidado para a vida diária como a alimentação, a mobilidade, a higiene, sem comprometimento cognitivo ou com alteração cognitiva moderada, e o grau de dependência III são os idosos que necessitam de assistência em todas as AVDs e ou com comprometimento cognitivo.

Dados apresentados pelas Nações Unidas (2007), apontam que o envelhecimento da população pode afetar os sistemas de cuidados de saúde de duas maneiras: em primeiro lugar, o aumento do número total de casos de doenças crônicas e o maior número de pessoas com deficiência, exigindo novas habilidades de profissionais e trabalhadores da área de saúde, das indústrias, dos designers e da sociedade de um modo geral, haja vista que essas novas demandas exigirão a criação de estrutura através de serviços e produtos que garantam a qualidade de vida dos idosos e de seus cuidadores. Isso representa um enorme desafio para os países em desenvolvimento com populações que estão envelhecendo rapidamente. Em segundo lugar, a preocupação sobre a forma como a prestação de cuidados de longa duração serão oferecidos àqueles cujas condições de saúde são irreversíveis, e cuja família não dispõem de condições para garantir-lhes o necessário. 


\section{GAMPI plural|15}

\section{O ATO DE CUIDAR}

$\mathrm{O}$ ato de cuidar é subjacente ao ser humano e pode ser uma experiência prazerosa ou estressante, dependendo do contexto e das capacidades ou incapacidades dos agentes envolvidos no ato de cuidar. O cuidador é quem tem a atitude de cuidar, ser diligente, zeloso e responsável pelo ser cuidado. Segundo Bonneterre e Santos (2008, web), o ato de cuidar é uma atitude e um comportamento em um relacionamento estabelecido que ocorrem em tipos e maneiras diferentes. Cuidar é um ato de preocupação, ocupação, responsabilização e envolvimento com o ser cuidado; pode ser considerado um ato de empatia, colocar-se no lugar do outro sentindo, compartilhando, sendo solidário e compreendendo o momento da outra pessoa.

O desenvolvimento dessas habilidades se torna essencial nesse cenário de envelhecimento populacional. Dependendo do grau de dependência ou incapacidade do idoso e pessoas com mobilidade reduzida, os cuidadores (formais ou informais) sofrerão uma carga emocional elevada, experimentando a tristeza, a impotência, frustração, culpa, raiva, medo, etc. É importante que o cuidador lide com esses sentimentos, pois podem acarretar efeito sobre a saúde física e mental, cujos sintomas podem ser depressão, estresse, imunidade baixa, etc.

Por outro lado, a experiência de cuidado pode trazer grande alegria e satisfação. Promover o bem-estar de outra pessoa, sendo responsável por zelar, proteger e amparar em qualquer situação, podem ser fatores motivacionais para minimizar o estresse gerado pelo ato de cuidar.

\section{O CUIDADO FAMILIAR}

Segundo o Estatuto do Idoso (2003), é obrigação da família, da comunidade, da sociedade e do poder público assegurar ao idoso o direito à vida, à saúde, à alimentação, à educação, à cultura, ao esporte, ao laser, ao trabalho, à cidadania e à liberdade.

A legislação brasileira estabelece que o cuidado dos idosos deva ser de responsabilidade das famílias, mas essa condição está se tornando escassa em função da redução da fecundidade, 


\section{GAMPI
plural' 15}

das mudanças na nupcialidade e da crescente participação da mulher no mercado de trabalho. A mulher é considerada tradicionalmente como a principal cuidadora (Camarano, 2010).

A capacidade dos familiares de cuidarem dos seus membros idosos está sendo afetada em virtude dessas mudanças estruturais acentuadas nas famílias. Hoje a mulher está tomando um papel mais ativo na família, dividindo a tarefa de provedor com o homem. Em 2009 ela foi responsável por mais de $40 \%$ da renda familiar. Percebe-se que a oferta de cuidado familiar diminui em face ao aumento da demanda.

A Constituição de 1988 explicita que apenas na impossibilidade de a família cuidar do idoso, instituições específicas devem ser consideradas uma alternativa de atendimento. Em virtude dessa situação, o Estado e o mercado podem dividir com a família a responsabilidade no cuidado da população idosa. Essa perspectiva traz uma alternativa de cuidado não familiar que corresponde às instituições de longa permanência para idosos (ILPIs), sejam essas públicas ou privadas (Camarano e Mello, 2010).

\section{O CUIDADO INSTITUCIONALIZADO}

A origem das instituições de longa permanência (ILPIs) está ligada aos asilos, que eram inicialmente dirigidos à população carente que necessitava de abrigo e assistência. Quando se fala em 'asilo', as primeiras imagens que vem a mente é a de abandono, desolação, prisão, maus tratos, pobreza, morte, etc.

A Anvisa define ILPI como governamentais e não governamentais, de caráter residencial, destinadas ao domicílio de pessoas idosas, com ou sem suporte familiar, em condições de liberdade, dignidade e cidadania (RDC No 283 de 26 de setembro de 2005). A ILPI pode ser definida como uma residência coletiva para atender idosos independentes em situação de carência de renda, de família e também aqueles com problemas para o desempenho das atividades da vida diária, que precisam de cuidados prolongados (Camarano, 2010).

A ILPI pode ser uma alternativa quando a família realmente não tem condições ou habilidades necessárias para cuidar e prover a qualidade de vida que o idoso necessita, independente do grau de dependência. Sejam elas públicas ou privadas, em face a esse contexto apresentado, podem ser uma alternativa de cuidado de longa duração, podendo gerar um 


\section{GAMPI
plural' 15}

benefício social na garantia da qualidade de vida dos idosos nas últimas fases da vida, assumindo parcialmente o papel das famílias que não sejam mais capazes ou estejam limitadas em prover a assistência adequada aos seus entes, devido a uma estrutura precária ou devido a situações de conflito.

\section{OS PROFISSIONAIS DA SAÚDE E OS PROBLEMAS OCUPACIONAIS CAUSADOS PELA MOVIMENTAÇÃO DOS PACIENTES}

Prestar cuidados aos idosos é um trabalho fisicamente exigente, muitas vezes necessitam de assistência para andar, tomar banho ou executar outras atividades do cotidiano. Em alguns casos, os pacientes são totalmente dependentes dos cuidadores para a realização das atividades da vida diária. Um exemplo é a elevação e movimentação manual, e outras tarefas que envolvem o reposicionamento, as quais estão associadas a um aumento do risco de lesões dos cuidadores, particularmente na região lombar.

Os fatores de risco que os cuidadores enfrentam são os seguintes (OSHA, 2009):

- Força - a quantidade de esforço físico necessário para executar uma tarefa (como o levantamento de peso) ou para manter controle de equipamentos ou ferramentas;

- Repetição - realizando o mesmo movimento ou série de movimentos continuamente ou frequentemente;

- Posturas inadequadas - posições que colocam pressão sobre o corpo, como chegar acima da altura do ombro, ajoelhado, cócoras, inclinando-se sobre uma cama ou torcendo o dorso ao levantar.

De acordo com a American Nurse Association (ANA), as tarefas de movimentação de pacientes são reconhecidas como a principal causa de distúrbios osteomusculares entre os trabalhadores de enfermagem. A preocupação principal são as lesões nas costas e tensões nos ombros que podem ser severamente debilitantes. Há uma variedade de tarefas de movimentação do paciente no contexto dos cuidados de enfermagem, tais como o levantamento, transferência e reposicionamento, que normalmente são executadas manualmente.

Owen \& Garg (1990) apud Nelson (2001), classificaram algumas tarefas em ordem de risco na movimentação de pacientes: 


\section{GAMPI
plural ${ }^{15}$}

1. Transferência de paciente da cama para a cadeira de rodas, da cadeira para vaso sanitário, da cadeira para cadeira de banho, da cama para a maca;

2. A transferência de paciente da banheira para a cadeira e vice-versa.

3. A transferência de paciente da cadeira para a cadeira do elevador;

4. Pesar o paciente.

5. Levantar o paciente na cama.

6. Reposicionamento de um paciente de um lado para o outro da cama.

7. Reposicionamento do paciente na cadeira.

Para amenizar e evitar as lesões são utilizados alguns equipamentos a fim de facilitar a transferência dos pacientes do leito, minimizando o impacto da disfunção física dos idosos sobre os cuidadores.

A pesquisa de campo apresentada neste artigo aborda a movimentação e transferência de pessoas com mobilidade reduzida no leito, considerando que isto afeta majoritariamente os idosos, mais suscetíveis à dependência e aos cuidados especiais.

\section{PESQUISA DE CAMPO}

O design é um processo pelo qual buscam-se soluções criativas para facilitar e melhorar a qualidade de vida das pessoas, resolvendo problemas do cotidiano e humanizando as tecnologias. Segundo Brown (2010), as pessoas devem estar no centro desse processo para ajudá-las a articular as necessidades que às vezes nem sabem que tem. A pesquisa realizada através da imersão do pesquisador no contexto de uso, pode desenvolver novas habilidades, inspirando a criatividade e a inovação para a criação de produtos, estratégias e serviços de valor.

O Design pode ser considerado como uma área de produção social, colocando-se como vital para constituição da riqueza, da geração de trabalho, de empregos e principalmente no desenvolvimento da sociedade, através da criação de produtos e serviços. É possível dizer que a criação nasce da sociedade, e daí trazer a discussão da influência do meio social na produção de bens materiais nesse processo (Cipiniuk, 2014). 


\section{GAMPIII $_{\text {plura }}$}

Este trabalho se caracteriza como uma pesquisa social com uma visão holística, no qual estão inseridos fatores contextuais, pessoais e de mobilidade, os quais são estudados para a definição do problema, embasando o desenvolvimento de soluções criativas.

A presente pesquisa é qualitativa, de cunho exploratório e aplicada. Os métodos qualitativos ajudam os pesquisadores a desenvolver empatia, permitindo uma abordagem próxima ao usuário final. Também permite o questionamento de suposições para inspirar novas soluções. (Human Centered Design - HCD - Tool Kit, 2014)

Segundo Gil (2008), a pesquisa aplicada caracteriza-se pelo interesse na aplicação, utilização e consequências práticas dos conhecimentos, preocupando-se na aplicação imediata numa realidade apresentada. A pesquisa aplicada depende das descobertas e se enriquece com o seu desenvolvimento. Esse tipo de pesquisa a que mais se dedicam os psicólogos, sociólogos, economistas, assistentes sociais e outros pesquisadores sociais onde pode-se incluir os designers.

A metodologia de desenvolvimento do projeto está baseada no processo de Design Thinking desenvolvido por Hasso Platner Institute of Design at Stanford University. É uma ferramenta prática para a solução de problemas conhecidos ou desconhecidos, dividida em cinco fases que podem acontecer de forma simultânea. Conforme demonstradas no quadro 1. 


\section{GAMPI}

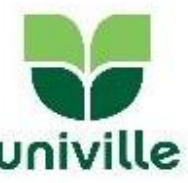

Quadro 1: Fases do processo de Design Thinking

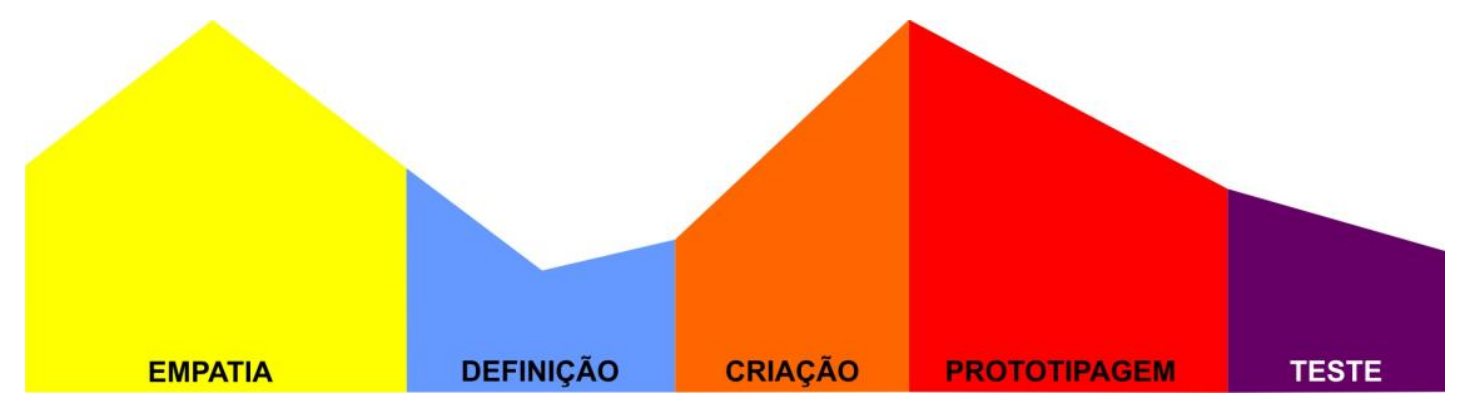

EMPATIA - Entender, observar, interagir e se relacionar com as pessoas para vivenciar a experiência de uso. Compreender através da perspectiva dos cuidadores as suas necessidades físicas e emocionais, como e porque as atividades são realizadas, o comportamento, e estabelecer relacionamentos, capturando o significado das experiências;

DEFINIÇÃO - É o processo de síntese das descobertas proveniente da fase de empatia. É necessário processar tudo o que foi visto e observado para entender as necessidades e definir o problema de forma clara para direcionar o processo da criação da solução;

CRIAÇÃ̃ - É a fase de geração de ideias, explorando e gerando uma ampla variedade de possíveis soluções;

PROTOTIPAGEM - É a transição da ideia para a forma física, transformando as propostas de solução do problema em artefatos concretos para experimentar e interagir fisicamente com a solução;

TESTE - Obter opiniões para refinar e validar a proposta selecionada.

Fonte: Adaptado pelo autor a partir de Hasso Plattner Institute of Design at Stanford.

$\mathrm{Na}$ fase da empatia utilizou-se duas técnicas de pesquisa, conforme quadro 2 :

Quadro 2: Técnicas de pesquisa

Entrevista individual: técnica realizada por meio de um questionário estruturado como investigação e forma de interação social, visando a obtenção de informações que interessam ao processo de pesquisa. Tem como o objetivo a coleta de dados voltada à orientação a cerca do que as pessoas sabem, creem, sentem e desejam a respeito do objeto de estudo (GIL, 2008).

Observação do participante: técnica etnográfica qualitativa pela qual o pesquisador acompanha, observa e vivência as atividades realizadas pelas pessoas no próprio contexto de uso. Desta maneira é possível interagir com os participantes da ação e com o ambiente. Esta técnica pode ser empregada nas fases iniciais da pesquisa e nas fases de prototipagem e testes. Estar no ambiente de trabalho ou doméstico das pessoas é delicado, é preciso ser discreto para que os participantes se sintam a vontade para serem autênticos na descrição das suas atividades e opiniões (MORAES \& SANTA ROSA, 2012).

Fonte: adaptado pelo autor com base em Gil, 2008 e Moraes \& Santa Rosa, 2012. 


\section{GAMPI
plural ${ }^{15}$}

A metodologia apresentada auxilia e direciona o processo de aprendizagem sobre o contexto dos profissionais da saúde e dos idosos nas ILPIs, contribuindo no processo de síntese das informações para a definição do problema e elaboração de hipóteses. A pesquisa preliminar fundamenta a criação de uma solução para melhorar a segurança física e emocional dos cuidadores e idosos.

A pesquisa de campo foi realizada em duas instituições de longa permanência para idosos, no Ventura Residence, situado na Avenida Procópio Gomes, nº 669 e na Casa de Repouso Pôr do Sol, localizado na rua Benjamin Constant, 851, ambas localizadas em JoinvilleSC.

Para vivenciar e experimentar o processo do ato de cuidar dos cuidadores nos ILPIs, a fase da empatia foi dividida em duas etapas:

- A primeira foi a realização das entrevistas individuais, tendo como base um questionário. As entrevistas foram presenciais e conduzidas no ambiente das instituições com cinco profissionais. No Ventura Residence foram entrevistadas quatro pessoas: uma enfermeira, a terapeuta ocupacional e uma fisioterapeuta. Na Casa de Repouso Pôr do Sol foram entrevistados o enfermeiro responsável pelo cuidado dos idosos e a proprietária da instituição.

- A segunda etapa foi a realização da observação do participante como técnica etnográfica, registrando uma parte da rotina por meio de imagens das atividades desempenhadas, para fornecer informações complementares as obtidas nas entrevistas. O registro das imagens se deu somente nos momentos de movimentação dos idosos com ou sem interação dos cuidadores dentro do ambiente, sem expor o paciente ou o cuidador a situação de risco físico ou constrangimento.

\section{A IMERSÃO}

As entrevistas individuais foram realizadas na semana anterior a imersão e os relatos dos profissionais apontaram a transferência e movimentação de pacientes no leito como a principal dificuldade ocupacional, acarretando desgaste físico e afastamentos do trabalho. 


\section{GAMPI \\ plural 15}

Através da imersão e observação da atividade, foi possível constatar que a movimentação e transferência de idosos no leito é uma atividade penosa com alto esforço físico, confirmando o que já havia sido mencionado nas entrevistas. A figura 1 e 2 demonstram o processo de transferência manual realizado pelos cuidadores no Ventura Residence e na Casa de Repouso Pôr do Sol respectivamente. 


\section{plural $^{\text {GA }}$}

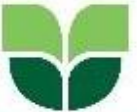 \\ univille}

Figura 1: Movimentação e transferência

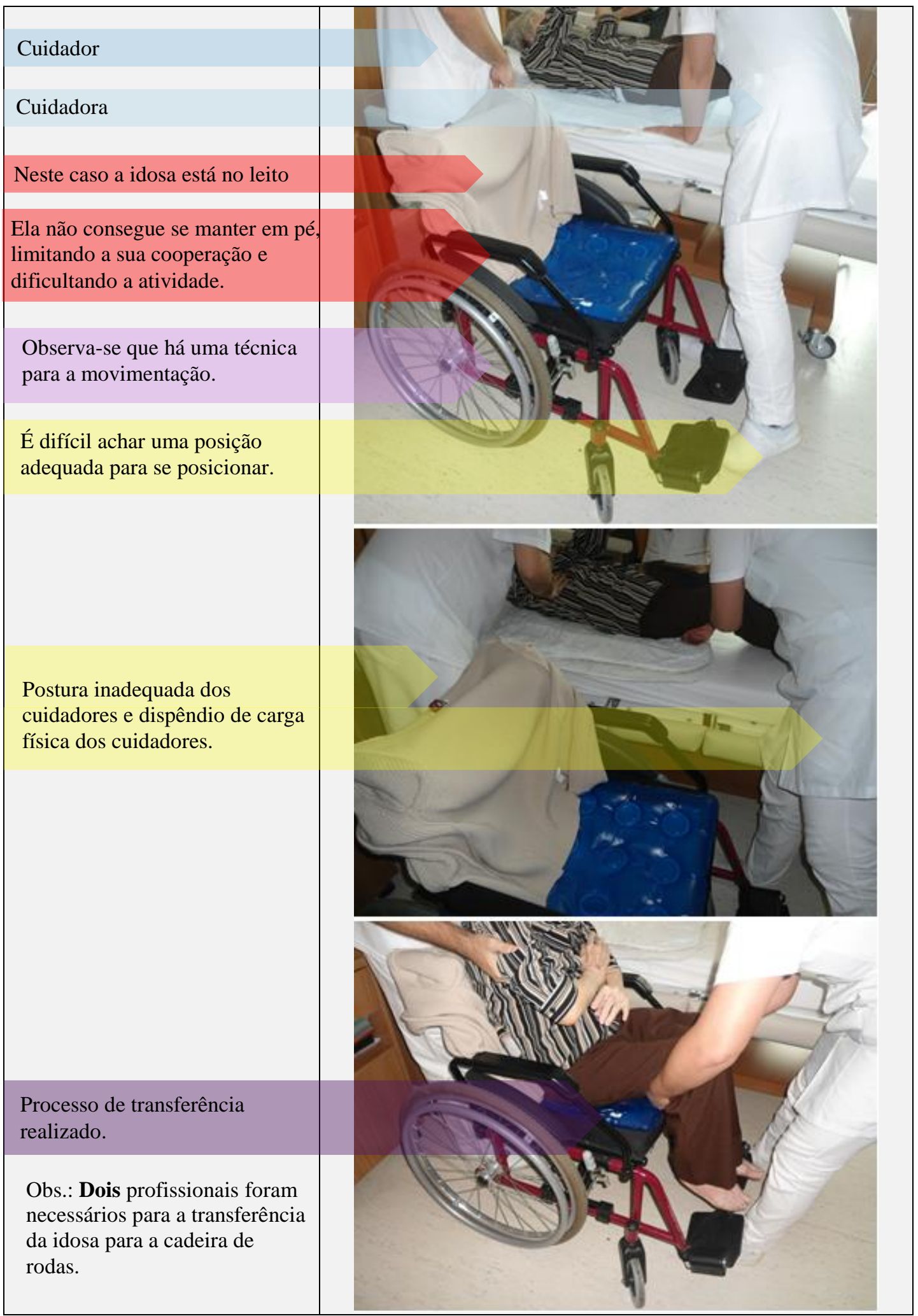

Fonte: Primária (2015). 


\section{GAMPI
plural ${ }^{15}$}

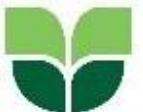 \\ univille}

Figura 2: Movimentação e Transferência

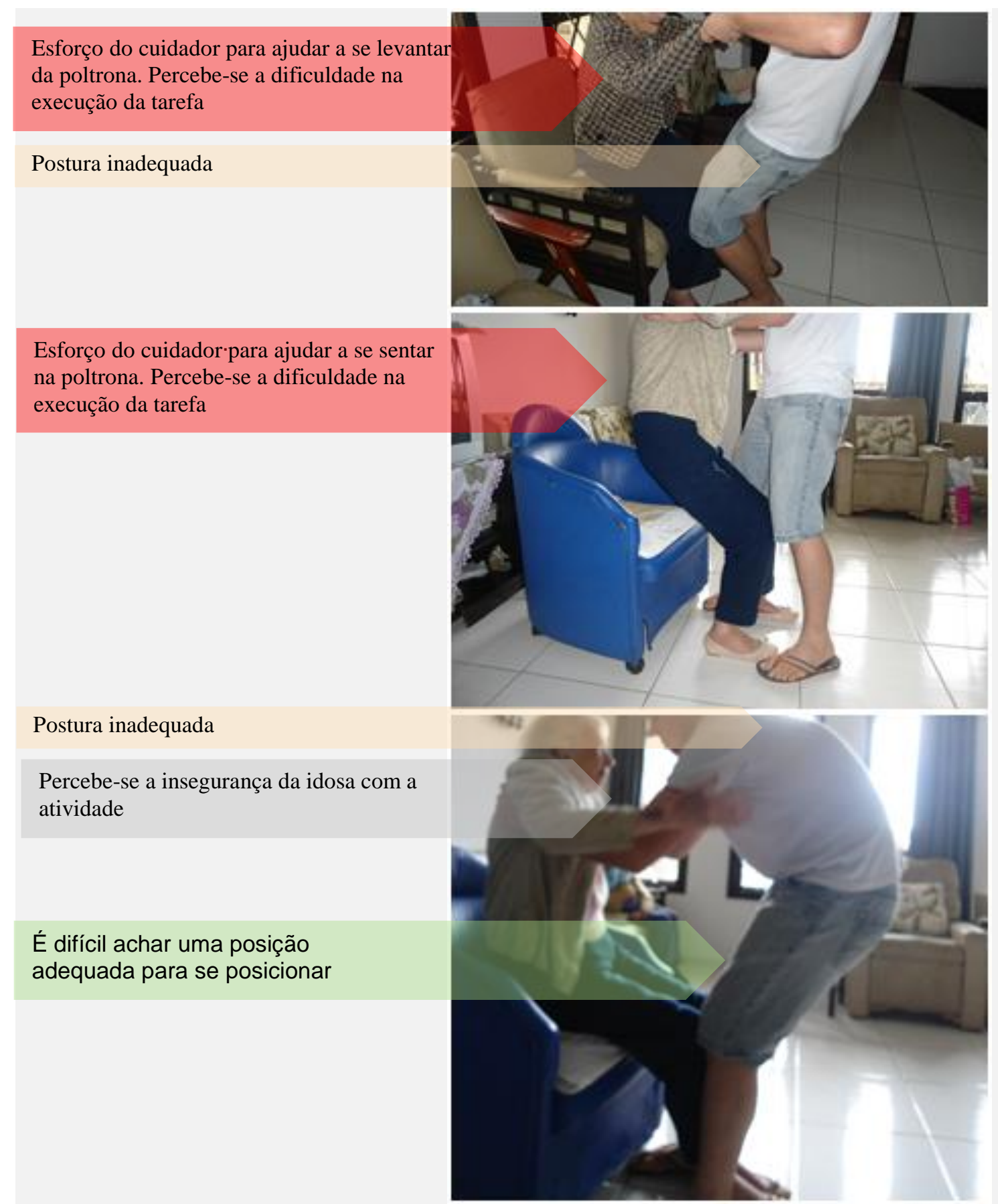

Fonte: Primária (2015).

Algumas tecnologias, estão disponíveis para os profissionais da saúde afim de garantir a assistência adequada para execução da atividade de movimentação e transferência. Em alguns casos é utilizado o elevador portátil (figura 3) para a transferência do paciente para a cadeira de 


\section{GAMPI plural 15}

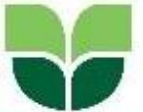 \\ univille}

rodas ou diretamente ao banheiro para as atividades de higiene, e para realizar qualquer tarefa de movimentação no leito.

Figura 3: Elevador portátil

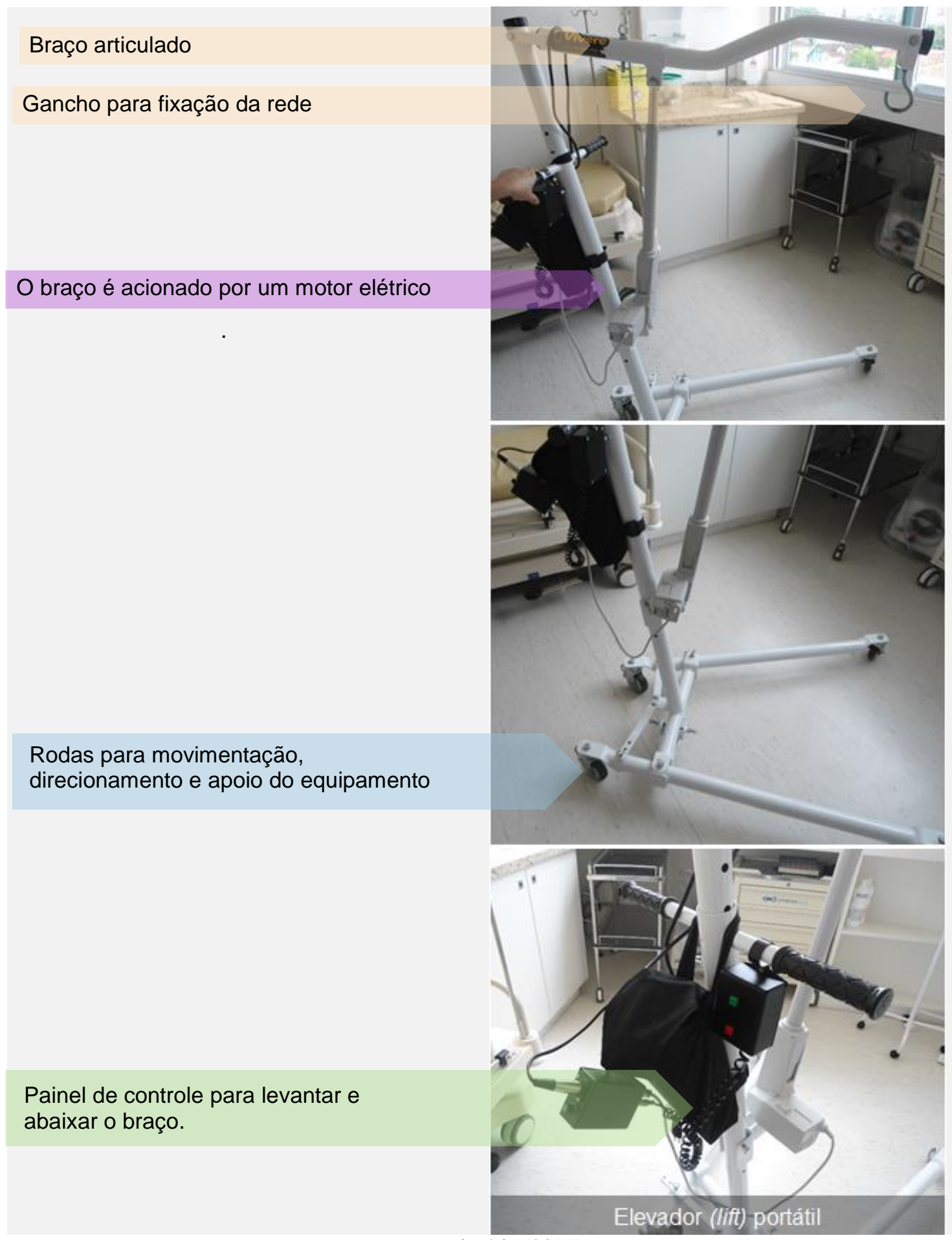

Fonte: Primária (2015). 


\section{plural $^{\text {GI }}$}

Apesar da disponibilidade dos equipamentos como o elevador portátil apresentado anteriormente, existem situações em que os cuidadores preferem fazer a transferência manual. Segundo relato dos profissionais, algumas pessoas rejeitam serem movimentados pelos equipamentos porque se sentem constrangidos e inseguros.

\section{CONCLUSÕES DA PESQUISA DE CAMPO}

A pesquisa de campo proporcionou uma experiência de aprendizado profundo sobre as questões acerca do ato de cuidar dos idosos nas instituições. Foi possível sentir a empatia vivenciando as dificuldades, frustrações, alegrias e os mais variados sentimentos nesse processo de imersão.

A diferença de estrutura física e recursos humanos e instrumentais das duas instituições chamou bastante a atenção durante a pesquisa. A figura 4 demonstra esse contraste acentuado e foi importante para evidenciar os pontos de intersecção da pesquisa nas duas instituições.

Figura 4: Ponto de intersecção: Ventura Residence e Casa de Repouso Pôr do Sol

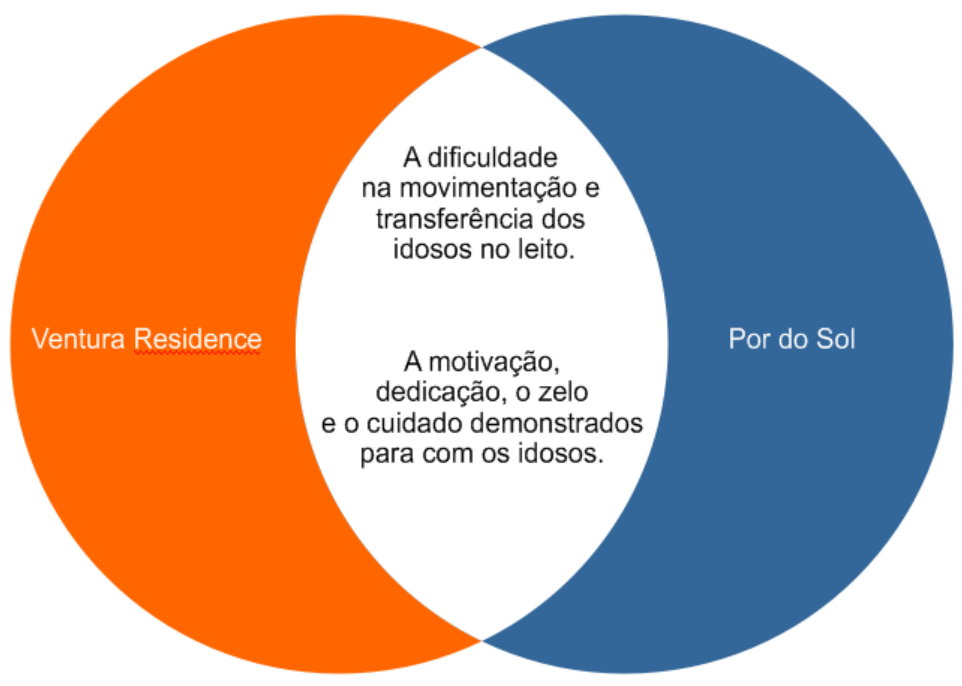

Fonte: Primária (2015)

Foi possível perceber que para garantir a qualidade de vida e o bem-estar dos idosos que vivem em instituições, deve-se valorizar o cuidador que é diretamente responsável por zelar, 


\section{plumPl $^{\text {GI }}$}

proteger e ampará-los nas atividades cotidianas (neste caso substituindo, em parte, o cuidado familiar).

Na movimentação e transferência de idosos manualmente, o cuidador assume posturas inadequadas, colocando pressão sobre o corpo e elevando o nível de esforço e o desgaste físico, podendo causar lesões musculares, desmotivando, frustrando, gerando afastamentos e comprometendo a qualidade da assistência. Mesmo quando recursos instrumentais necessários estão disponíveis, ainda há dificuldades na sua utilização. Verificou-se nas entrevistas que os profissionais da saúde em geral têm acesso, na maioria das vezes, somente à produtos inadequados, não atendendo plenamente as necessidades de uso.

As entrevistas e observações revelaram que há poucas opções e custo elevado dos produtos para auxílio desta tarefa, sendo muitos deles importados.. Bons produtos, com boas tecnologias aplicadas são caros, não estão disponíveis no país e o custo da importação pode inviabilizar a aquisição no Brasil. Desta maneira, abre-se caminho para produtos adaptados, inadequados e paliativos, tecnologias sem os atributos necessários para garantir a eficácia, a dignidade, a segurança e o conforto físico dos cuidadores e idosos.

Os problemas de transferência descritos na teoria foram comprovados na prática com a imersão nas ILPI's. Os dados são válidos para suportar o processo do design afim de buscar uma solução criativa para o problema identificado, melhorando as condições para realização desta tarefa. Desta forma, o contexto apresentado valida, direciona e embasa o desenvolvimento de uma solução (tecnologia) para auxiliar na atividade de movimentação e transferência dessas pessoas com grau de dependência II1 no leito, que possuem capacidade de cooperar com o cuidador. A solução deve ser baseada nas necessidades dos envolvidos neste processo para evitar os problemas físicos e emocionais gerados por essa atividade.

A partir da pesquisa de campo realizada, surge o seguinte questionamento de pesquisa: como propor uma solução segura e eficaz para movimentar e transferir pessoas com mobilidade reduzida no leito, sem constrange-los e reduzir as sequelas geradas pelo trabalho dos profissionais nesta atividade?

\footnotetext{
${ }^{1}$ Grau de dependência II são os idosos com dependência de até três atividades de autocuidado para a vida diária com a alimentação, a mobilidade, a higiene, sem comprometimento cognitivo ou com alteração cognitiva moderada.
} 


\section{GAMPIII $^{\text {GA }}$}

\section{CONSIDERAÇÕES FINAIS}

O design busca criar soluções criativas e eficazes para facilitar a vida das pessoas ao resolver problemas do dia-a-dia, tornando as tecnologias mais acessíveis. Segundo Cipiniuk (2014), limitar o design apenas à dimensão estética, ou mesmo à noção funcionalista, pode reduzir a sua importância e a sua necessidade para a sociedade como um todo. Acredita-se que a solução de design a ser proposta nesta pesquisa aplicada seja desejável, viável, factível, proporcionando benefícios à sociedade e suprindo a escassez de produtos para auxiliar na movimentação e transferência no leito das pessoas com mobilidade reduzida. A solução pretende atender às necessidades e desejos dos atores envolvidos, cuja proposta de valor seja inovadora e baseadas nos princípios de design, na ergonomia e usabilidade afim de facilitar o ato de cuidar.

Para alcançar os objetivos propostos nesta pesquisa aplicada em andamento, se faz necessário a execução das atividades descritas na metodologia. Até o presente momento, as fases de empatia e definição foram concluídas e foram apresentadas na qualificação do Mestrado Profissional em Design da Univille, e resumidas neste artigo.

\section{Referências}

ANA (Amarican Nurses Association). Handle With Care Fact Sheet. Disponível em: http://www.nursingworld.org/MainMenuCategories/WorkplaceSafety/Healthy-Work-

Environment/SafePatient/Resources/FactSheet.html. Acesso em: 20/06/2015;

BONETTERRE, Adriana; SANTOS, Sandra. Cuidando de quem cuida. Web, 2008. Disponível em: http://psiclinicaehospitalar.blogspot.com.br/2008/03/cuidando-de-quemcuida-o-que-significa.html. Acesso em 25/06/2015;

BRASIL. Decreto 3.956 de 8 de outubro de 2001. Promulga a Convenção Interamericas para a Eliminação de Todas as Formas de Discriminação contra Pessoa Portadoras de Deficiência. Disponível em: http://portal.mec.gov.br/seesp/arquivos/pdf/guatemala.pdf. Acesso em 20/11/2014;

BROWN, Tim. Design Thinking. $3^{\text {a }}$ ed. Rio de Janeiro: Elsevier, 2010; 


\section{GAMPI
plural ${ }^{15}$}

CAMARANO, Ana Amélia. Cuidados de longa duração para a população idosa: família ou instituição de longa permanência? Sinais Sociais, 2008. Disponível em: http://www.sesc.com.br/portal/publicacoes/sesc/revistas/sinaissociais/n20/n: Acesso em: 20/05/2015;

CAMARANO, Ana Amélia; Kanso, Solange. As Instituições de longa permanência no Brasil. Revista Brasileira de Estudos Populacionais: Rio de Janeiro, 2010. Disponível em: http://www.scielo.br/pdf/rbepop/v27n1/14.pdf. Acesso em 5/06/2010;

CAMARANO, Ana Amélia; KANSO, Solange. Instituições de longa permanência para idosos: Abrigo ou retiro? In: CAMARANO, A. A. (Org.). Cuidados de longa duração para a população Idosa: um novo risco assumido? Rio de Janeiro: Instituto de Pesquisa Econômica Aplicada - IPEA, 2010. p. 164.

Como as famílias estão lidando com idosos que demandam cuidados e quais as perspectivas futuras? In: CAMARANO, A. A. (Org.). Cuidados de longa duração para a população Idosa: um novo risco assumido? Rio de Janeiro: Instituto de Pesquisa Econômica Aplicada - IPEA, 2010. p. 113;

CAMARANO, Ana Amélia; at al. As instituições de longa permanência para idosos no Brasil In: CAMARANO, A. A. (Org.). Cuidados de longa duração para a população Idosa: um novo risco assumido? Rio de Janeiro: Instituto de Pesquisa Econômica Aplicada - IPEA, 2010. p. 190.

CAMARANO, Ana Amélia; MELLO, Juliana Leitão. Introdução In: CAMARANO, A. A. (Org.). Cuidados de longa duração para a população Idosa: um novo risco assumido? Rio de Janeiro: Instituto de Pesquisa Econômica Aplicada - IPEA, 2010.

CIPINIUK, Alberto. Design: O livro dos porquês: o campo do Design compreendido como produção social. Rio de Janeiro: ED. PUC-RIO; São Paulo: Ed. Reflexão, 2014.

CHRISTOPHE, Micheline. Instituições de longa permanência para o idoso no Brasil: uma opção de longa duração? 2011. Dissertação de Mestrado - Escola Nacional de Ciências Estatístticas. Rio de Janeiro;

GIL, Antonio Carlos. Métodos e Técnicas de Pesquisa Social. 6 ed. São Paulo. Editora Atlas, 2008;

HCD. Kit de Ferramentas. 2 ed: Ideo. Disponível em: http://www.ideo.com/images/uploads/hcd_toolkit/HCD_Portuguese.pdf. Acesso em 15/08/2004; 


\section{GAMPI}

PLATTNER, Hasso. An Introduction to Design Thinking. Process Guide. Institute of Design at Stanford. Disponível em: https://dschool.stanford.edu/sandbox/groups/designresources/wiki/36873/attachments/74b3d/ ModeGuideBOOTCAMP2010L.pdf?sessionID=9a5d0a2a0cd5fb6c26a567b2636b19513b76d Of4. Acesso em: 15/05/2015;

OSHA (Occupational Safety an Health Administration). Guidelines for Nursing Homes Ergonomics for the Prevention of Musculoskeletal Disorders. U.S. Departamento of labor, 2009. Disponível em: www.osha.gov. Acesso em 19/06/2015;

SAMPAIO, Aline Melo Oliveira, et al. Cuidadores de idosos: percepção sobre o envelhecimento e sua influência sobre o ato de cuidar. Rio de Janeiro. 2010. Disponível em: http://pepsic.bvsalud.org/scielo.php?script=sci_arttext\&pid=S1808-42812011000200015.

Acesso em: 05/06/2015;

SECRETARIA DE DIREITOS HUMANOS. Dados sobre o envelhecimento no Brasil. Disponível em: http://www.sdh.gov.br/assuntos/pessoa-idosa/dadosestatisticos/DadossobreoenvelhecimentonoBrasil.pdf. Acesso em 20/04/2015.

WHO (World Health Organization). WHO Global Forum of Innovations for Ageing Population, 2013. Disponível em: http://www.who.int/kobe_centre/publications/gfiap_report/en/. Acesso em: 15/06/2015; 